\title{
Practice-related reduction of dual-task costs under conditions of a manual-pedal response combination
}

\author{
Roman Liepelt ${ }^{1}$, Rico Fischer ${ }^{2}$, Peter A. Frensch ${ }^{3}$, and Torsten Schubert ${ }^{4}$ \\ ${ }^{1}$ Department of Psychology, Westfälische Wilhelms-University, Münster, Max Planck \\ Institute for Human Cognitive and Brain Sciences, Department of Psychology, Leipzig, \\ and Humboldt-University, Berlin, Germany \\ ${ }^{2}$ Humboldt-University, Berlin, and Technische Universität, Dresden, Germany \\ ${ }^{3}$ Humboldt-University, Berlin, Germany \\ ${ }^{4}$ Ludwig-Maximilians University, München, and Humboldt-University, Berlin, Germany
}

\begin{abstract}
Severe dual-task costs emerge when two tasks are performed at the same time. Schumacher, Seymour, Glass, Kieras, and Meyer (2001) showed a complete reduction of dual-task costs after extensive dual-task practice with a visual-manual (VM) task and an auditory-verbal (AV) task. First, we replicated these findings and found task conditions sufficient to achieve a high level of dual-task cost reduction (Experiment 1). Using these conditions, we tested whether the Schumacher et al. findings generalise to a different dual-task situation, in which participants practised a VM task and an auditory-pedal (AP) task (VM-AP) conjointly (Experiment 2). In the VM-AP task situation we found reduced dual-task costs after practice. Dual-task costs, however, remained on a high level after eight sessions of practice and also when extending practice to 12 sessions. No single participant showed evidence for time sharing in the VM-AP dual task. These results suggest that the finding of complete dual-task cost reduction does not generalise to the VM-AP task combination used in the present study. We discuss different factors potentially relevant for the observation of persisting dual-task costs over practice in the VM-AP task.
\end{abstract}

Keywords: Dual task; Manual-pedal; Practice.

Observations from everyday life and many findings in psychological research show that severe dual-task costs emerge when two tasks are performed at the same time (Lien \& Proctor, 2002; Pashler \& Johnston, 1989). For example, the simultaneous execution of two choice-reaction tasks leads to dramatic slowing in one (Pashler, 1994) or in both component tasks (Tombu \& Jolicoeur, 2004) compared to a single-task situation. Such performance decrements in dual-task situations compared to single-task situations are known as "dual-task costs" and have often been attributed to structural processing limitations at the response selection stage (Pashler, 1994; Pashler \& Johnston, 1989; Schubert, 1999) and/or the response initiation stage (de Jong, 1993; Meyer \& Kieras, 1997a, 1997b).

A typical explanation for dual-task costs is that the response selection stage in one task is postponed until the processing of the response selection stage in the other task is completed because of resource limitations within the processing

Correspondence should be addressed to Roman Liepelt, Westfälische Wilhelms-University, Department of Psychology, Fliednerstrasse 21, 48149 Münster, Germany. E-mail: roman.liepelt@uni-muenster.de

The present research was supported by a grant of the German Research Foundation to TS and PF (DFG Schu 1397/ 3-2). The authors wish to thank Mei-Ching Lien and Eric Ruthruff for helpful comments on a previous version of the manuscript. We are grateful to Franziska Plessow and Marina Palazova for their help in collecting data. 
stream (for reviews see Meyer \& Kieras, 1997a, 1997b; Pashler, 1994).

Recent studies provided evidence that extensive dual-task practice may result in a complete reduction of dual-task costs (Hazeltine, Teague, \& Ivry, 2002; Schumacher, Seymour, Glass, Kieras, \& Meyer, 2001; see also Spelke, Hirst, \& Neisser, 1976, for an earlier study). For example, Schumacher et al. (2001) trained participants over five learning sessions ( 1 hour per day) to respond to the horizontal location of an "O" with right-hand finger keypresses. At the same time, participants were required to respond verbally to the frequency of a tone. In this particular combination of a visual-manual (VM) and an auditory-verbal (AV) task, dual-task costs were calculated as the difference between dual-task reaction times (RTs) and single-task RTs. Whereas large dualtask costs were found at the beginning of learning (Session 2), these costs were completely eliminated by the end of learning (Session 5). Such findings of eliminated dual-task costs after practice are important because they may provide important insights about the mechanisms and conditions for practice-related changes in dualtask performance. For example, proponents of the response selection assumption explain similar observations by assuming a latent bottleneck after practice (Ruthruff, Johnston, van Selst, Whitsell, \& Remington, 2003; van Selst, Ruthruff, \& Johnston, 1999). Accordingly, a structural limitation at the response selection stage may still be intact after practice but the bottleneck stages may be extremely shortened. This shortening reduces the overlap of critical processing stages and may eventually eliminate a significant or detectable postponement in one of the two tasks (Ruthruff et al., 2003).

On the other hand, findings like those of Schumacher et al. (2001) are perfectly in line with dual-task models that account for dual-task costs by strategic rather than structural postponements at the central response selection stage (Meyer \& Kieras, 1997a, 1997b, 1999). These models assume that participants adopt specific processing strategies to optimise task performance during practice. According to Meyer and Kieras (1997a, 1997b), structural limitations are not related to response selection but instead to physical constraints of the peripheral motor systems involved in task processing (e.g., bimanual responses). From this perspective, the strategic postponement model claims that multiple actions can, in principle, be planned and selected concurrently (i.e., parallel response selection) after practice (see also Byrne \& Anderson, 2001). At the same time, Meyer and colleagues proposed a set of criteria that have to be fulfilled allowing participants to show perfect time sharing (e.g., changing task scheduling strategies in a way that parallel response selection is enabled). The first criterion asks for different motor effectors in both tasks. This rests on the assumption that any structural limitation is located at the response initiation or motor stage (de Jong, 1993; Karlin \& Kerstenbaum, 1968; Keele, 1973). Second, participants need to be given a sufficient amount of task practice. Most studies on perfect time sharing could show that at least 5-8 sessions of practice are required for sufficient dual-task cost reduction (Hazeltine et al., 2002, Schumacher et al., 2001; Tombu \& Jolicoeur, 2004). Participants might, however, lose their motivation due to the enormous length of practice. To keep motivation high during practice, Meyer and Kieras (1997a) proposed monetary reward that is directly performance related. Third, the task instruction should emphasise both tasks equally. Note that this is different from typical dual-task studies with temporal stimulus onset variations, in which Task 1 processing is typically emphasised over Task 2 processing. This restrictive task instruction might prevent participants to apply processing strategies that allow for time sharing. Even presenting one stimulus before the other might imply a priority for the task presented first. Therefore, studies providing an equal task emphasis via an equal priority instruction, as the Schumacher et al. (2001) study, additionally presented both stimuli at the same time with a zero stimulus-onset asynchrony (SOA). Because individual changes of task scheduling strategies seem to enable dual-task cost reduction and parallel response selection processing during practice findings of dual-task cost reduction and bottleneck elimination are related to the use of these task conditions (Schumacher et al., 2001). In the Schumacher et al. study, all these criteria are perfectly matched using a VM and an AV task, respectively.

Because findings about the practice-related reduction of dual-task costs represent a serious challenge for contemporary dual-task models it is important to test the conditions and the generalisability of time sharing. However, to our knowledge, most studies that have demonstrated virtual or complete reductions of dual-task costs after practice have relied on task combinations 
including either components of different modalities or used different effector systems such as manual and verbal motor responses (e.g., Hazeltine, Ruthruff, \& Remington, 2006; Hazeltine et al., 2002; Schumacher et al., 2001; Tombu \& Jolicoeur, 2004; but see Hirst, Spelke, Reaves, Caharack, \& Neisser, 1980).

Many interfaces for human-machine interaction, however, not only require responses with manual and verbal outputs, but use other types of output combinations, such as manual and pedal output combinations that require responses with hands and feet (e.g., driving a car). Dual-task situations involving manual-pedal pairings, for example, constitute separate modality systems that use separate motor processors. This avoids structural and peripheral motor interference, such as the situation in the Schumacher et al. (2001) study.

However, manual-pedal output combinations seem to contain a spatial dimensional overlap concerning the left-right dimension of hands and feet. Creating a dual-task situation with distributed responses in manual-pedal output combinations might therefore introduce crosstalk effects, on the basis of spatially mediated responseresponse (R-R) compatibility effects (e.g., Hommel, 1998; Koch \& Prinz, 2002; Lien \& Proctor, 2002; Logan \& Gordon, 2001; Schubert, Fischer, \& Stelzel, 2008). This kind of crosstalk might help to reduce dual-task costs under compatible R-R conditions (e.g., left-hand and left-foot responses), but hinder dual-task cost reduction under incompatible conditions (e.g., left-hand and right-foot response, respectively). Only little research has tested whether the finding of complete dual-task cost reduction after extensive dual-task practice (Schumacher et al., 2001) generalises to dual-task task situations involving manual-pedal pairings.

To our knowledge, only one recent study, which aimed to localise practice effects in dualtask performance, used a combination of manual and pedal motor responses (Sangals, Wilwer, \& Sommer, 2007). More specifically, the study combined a visual-manual and an auditory-pedal task as Task 1 and Task 2, respectively. Participants performed both tasks on five consecutive dual-task sessions (one per day), and thus received five sessions of practice. Although the authors found a reduction of dual-task costs over practice, considerable dual-task costs remained in both tasks after practice. The lack of time sharing in the Sangals et al. (2007) study seems astonishing at first, because they used task pairings that involve separate output processors. However, the Sangals et al. study differed to other studies, showing evidence for time sharing, in some important methodological aspects. For example, the Sangals et al. (2007) study used a typical psychological refractory period (PRP) paradigm (Pashler, 1994) in which stimuli of both tasks were presented sequentially with a variable SOA. The task instruction placed priority on Task 1 processing instead of using an equal task priority. In addition, task performance was only weakly rewarded by bonus points instead of monetary payoffs. This leaves the question open whether dual-task combinations using manual and pedal motor responses are quite resistant to practice or whether resistant dual-task costs in previous studies (Sangals et al., 2007) are due to strategies that participants adopted to the specific task conditions (Meyer \& Kieras, 1997a, 1997b) used in that study.

The aim of the present study was to test whether dual-task costs can be eliminated during practice when combining a visual-manual with an auditory-pedal (AP) task (Sangals et al., 2007) using a non-PRP design and applying the Meyer and Kieras (1997a, 1997b) criteria for dual-task cost reduction. If a complete dual-task cost reduction can be found for a manual-pedal dualtask pairing carefully applying the criteria of Meyer and Kieras, then the finding of dual-task cost reduction depends on the specific criteria applied and not on the manual-pedal pairing used. If under these conditions high and resisting dual-task costs remain after practice other sources of interference preventing a successful dual-task cost reduction need to be considered (see, for example, Hazeltine et al., 2006).

Experiment 1 of the present study served the purpose to test whether the criteria of Meyer and Kieras (1997a, 1997b) used in the present study are sufficient to find dual-task cost elimination using the same VM-AV task as in the Schumacher et al. (2001) study. In addition to the basic analyses of dual-task costs, we aim to check for dual-task cost elimination that can be achieved with practice on the individual-subject level.

\section{EXPERIMENT 1}

A range of studies obtained rather diverse results when investigating practice effects on dual-task cost reduction after having changed the SOA 
condition and the monetary reward system (cf. Hazeltine et al., 2002; Sangals et al., 2007; Tombu \& Jolicoeur, 2004). In Experiment 1, we used a task pairing that involved essentially the same task conditions (i.e., VM-AV task pairings with an SOA of $0 \mathrm{~ms}$ and monetary reward) as in the Schumacher et al. (2001) study. In line with Tombu and Jolicoeur (2004), we used a monetary reward system in which reward was calculated separately for dual-task and single task RTs.

\section{Methods}

Participants. Eight undergraduate students (2 male, age 21-26, mean age: 22.8 years) of Humboldt-University, Berlin, took part in the experiment. All participants had normal or corrected-to-normal vision, and were naive about the hypotheses of the experiment. All participants gave their written informed consent to participate in the study, which was conducted in accordance with the ethical standards of the 1964 Declaration of Helsinki.

Apparatus and stimuli. Stimuli were presented on a 17-inch colour monitor that was connected to a Pentium I PC. Experiments were carried out using Experimental Runtime System software (ERTS; Beringer, 1987-2000).

In the visual task, a white circle appeared at one of three possible locations (left, middle, or right). Participants responded manually in a spatially compatible mapping with the index, middle, or ring finger of their right hand. The circles were horizontally arranged on a black background of a computer monitor. Each circle subtended approximately $2.5 \mathrm{~cm}$, which corresponds to $2.38^{\circ}$ of visual angle by a viewing distance of $60 \mathrm{~cm}$. Three white lines served as placeholders and signalled the beginning of a trial. The distance between the circles was $1 \mathrm{~cm}$, which corresponds to approximately $0.95^{\circ}$. The three circles spread over a distance of $8.99^{\circ}$ and were presented until the participant responded or until a maximum response time of $2000 \mathrm{~ms}$ was reached. The responses were recorded with a response board connected to the computer.

For the auditory task, participants were asked to speak out loud "one" to a low frequent tone $(350 \mathrm{~Hz})$, "two" to a middle frequent tone $(900 \mathrm{~Hz})$, and "three" to a high frequent tone $(1650 \mathrm{~Hz})$. Verbal responses were recorded with a Sony microphone connected to a voice key.

Procedure and design. A trial started with white lines serving as placeholders signalling the beginning of a trial presented for $500 \mathrm{~ms}$. After this period a circle appeared in the visual task and remained visible until the participant responded or until a maximum of $2000 \mathrm{~ms}$ had expired. In the auditory task, a tone was sounded after $500 \mathrm{~ms}$ lasting for $40 \mathrm{~ms}$. RTs were given as feedback after each trial for $1500 \mathrm{~ms}$ followed by a blank screen for $700 \mathrm{~ms}$ (see Figure 1).

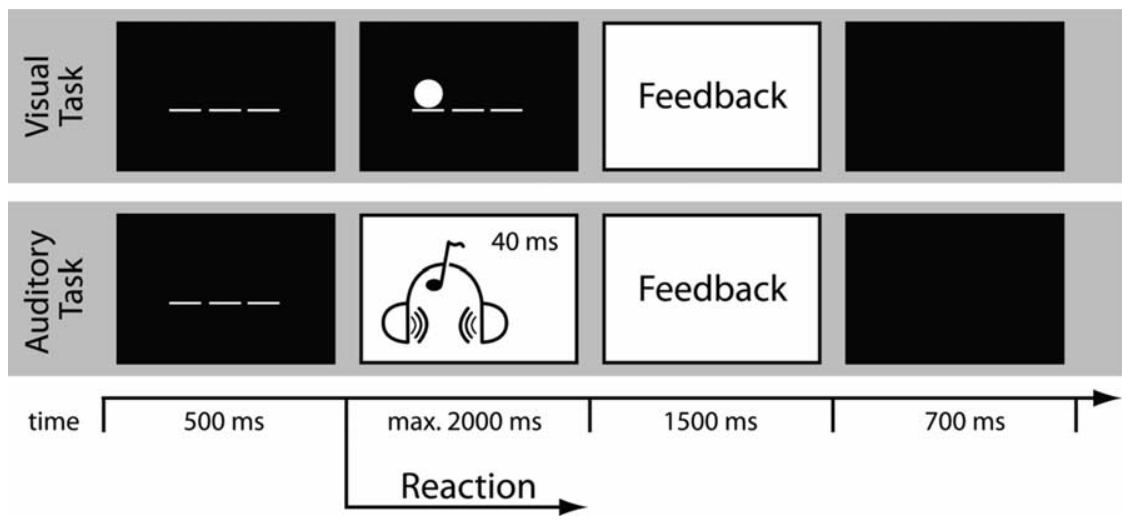

Figure 1. Stimulus sequence for each trial for the visual task (upper panel) and the auditory task (lower panel) in Experiment 1. Both trials started with a warning signal (displayed for $500 \mathrm{~ms}$ ) showing three lines which served as placeholders. Subsequently, a circle appeared in the visual task and remained visible until the participant responded or until a maximum of $2000 \mathrm{~ms}$ had expired (imperative response stimulus for the visual task). In the auditory task after the warning signal a tone was played for $40 \mathrm{~ms}$ (imperative response stimulus for the auditory task) and remained visible until the participant responded or until a maximum of $2000 \mathrm{~ms}$ had expired. RTs were given as feedback after each trial for $1500 \mathrm{~ms}$ followed by a blank screen for $700 \mathrm{~ms}$, which remained until the next trial started. 
The procedure was comparable to that used by Schumacher et al. (2001). There were two types of blocks, single-task blocks and mixed blocks. In the single-task blocks, participants performed 45 single-task trials. These could be either visualtask trials or auditory-task trials. During mixed blocks, participants performed 30 trials of either the visual task or the auditory task alone ("OR" trials) randomly intermixed with 18 "dualtask" trials. It should be noted that the term "OR" trials originally referred to blocks of trials in which either task could be required, but never both tasks (Schumacher et al., 2001). In the present study, with OR, we refer to trials in which either task could be required mixed in one block with dual-task trials. In dual-task trials, the two tasks were presented simultaneously with a zero SOA.

Participants were instructed to respond to both stimuli as quickly and accurately as possible during all blocks, to fully concentrate and to give equal priority to the two tasks. They were free to respond in any order. After each trial we gave the respective RT as feedback. To minimise the load of the feedback information during the ongoing trial, in dual-task trials, only the faster response time was given as feedback. When participants committed an error, the RT feedback was replaced by the German word for error ("Fehler") for the same amount of time.

Reward was given in the form of a monetary performance-based payoff to maximise participants' motivation for achieving accurate and fast performance (see also Schumacher et al., 2001; Tombu \& Jolicoeur, 2004). The payoff matrix was based on an adaptive comparison between participant's performance in a given trial (i.e., current RT) and a reference RT, the so-called target time. The experiment started with a target time of $2000 \mathrm{~ms}$, which was then adjusted after each block separately for each participant and task condition (single- vs. dual-task condition). Target times represented the mean RT of single-task trials in a single-task block and the mean RT of dual-task trials in a mixed block. OR trials played no role in the bonus payment.

Based on their personal performance improvement participants could earn more or less money. When participants' mean RT for a given block was slower than the target time, but still in a range of $50 \mathrm{~ms}$ to $100 \mathrm{~ms}$ above the target time, they received 10 cents in addition for that block. When the mean RT was in a range of $0 \mathrm{~ms}$ to $50 \mathrm{~ms}$ above the target time, they received 25 cents.
Importantly, when the RT of the ongoing block was faster than the target time, they received 50 cents and the RT of the ongoing block served as the new target time for the upcoming blocks. Mean RT of the current block and target time were presented at the end of each block.

Bonus payments were also made on the basis of accuracy rates: One additional cent was given for each correct response and 5 cents were deducted for each incorrect response. Participants earned separate bonuses for the two tasks (visual and auditory) as well as for single and mixed blocks. To increase motivation and task performance, the experimenter additionally encouraged participants verbally between blocks to respond as fast and accurate as possible.

In accordance with the study of Hazeltine et al. (2002), we started with a practice period of eight practice sessions ( 1 hour per day) in both conditions. In Session 1, participants performed 12 (six visual and six auditory) single-task blocks consisting of 45 single trials each. Session 2 included six single-task blocks (three visual and three auditory) and eight mixed blocks. The design in Sessions 3-8 was identical to that in Session 2 but included 10 mixed blocks in addition to the six single-task blocks (three visual and three auditory).

Visual and auditory task blocks were presented in an alternating order in Session 1. The block order in Sessions 2-8 was as follows: The first two blocks of Session 2 were single-task blocks (one visual and one auditory) and the next two blocks were mixed blocks. Following these four blocks, each single-task block was followed by two mixed blocks. Half of the participants started with a visual single-task block and the other half with an auditory single-task block in each session. The procedure and the structure of blocks and trials was the same for both conditions.

Altogether, the structure of the experiment can be described by: Session 1: $12 \times($ single task $)=540$ trials; Session 2: $6 \times($ single task $)$, $8 \times(\operatorname{mix})=654$ trials; Sessions 3-8: $6 \times($ single task $), 10 \times(\mathrm{mix})=4500$ trials. Participants performed 5694 trials in total.

\section{Results}

Prior to statistical RT analyses, all trials in which responses were incorrect were excluded (6.6\%). We used a two-factorial within-subject design containing the seven-level factor practice (Sessions 2-8) and the three-level factor trial 
type consisting of single-task trials, OR trials, and dual-task trials. Each task was analysed individually. Session 1 was considered as practice. For all repeated-measures ANOVAs, we corrected the $p$ values using Greenhouse-Geisser adjustments if necessary. In order to use a strong and reliable criterion for measuring dual-task costs, we measured dual-task costs in two different ways. First, we compared RTs in dual-task trials to those in single-task trials (dual minus single) (Tombu \& Joliceour, 2004). When speaking about dual-task costs in general we use this relatively strong criterion. Second, in line with Hazeltine et al. (2002), we additionally compared RTs between dual tasks and OR trials (dual minus OR). These analyses of dual-task costs are confined to Session 2 (beginning of learning) and Session 8 (end of learning). These analyses are performed by means of planned one-tailed t-tests.

Reaction time analysis of the visual task. Figure 2 (upper panel) shows the RTs for the visual task and for the auditory task as a function of Practice and Trial type (for corresponding error rates see Table 1). Participants responded faster with increasing practice, as reflected by the main effect of practice, $F(6,42)=11.34, M S E=1299.03$, $p<.001$. RTs in single-task trials were faster than RTs in OR trials, which in turn were faster than dual-task RTs, as indicated by the significant effect of trial type, $F(2,14)=11.32$, $M S E=2602.74, \quad p<.01$. Most importantly, we observed a significant interaction of practice $\times$ trial type, $F(12,84)=4.89, \quad M S E=242.25$, $p<.001$, indicating that practice reduced dualtask costs.

In Session 2, we found dual-task costs of $96 \mathrm{~ms}$, $t(7)=3.64, p<.01$ (dual minus single). Performance between dual-task and OR trials (Hazeltine et al., 2002) also differed in Session 2 by $46 \mathrm{~ms}, t(7)=2.89, p<.05$ (dual minus OR). Performance between dual-task and single-task trials in the visual task did not differ statistically in
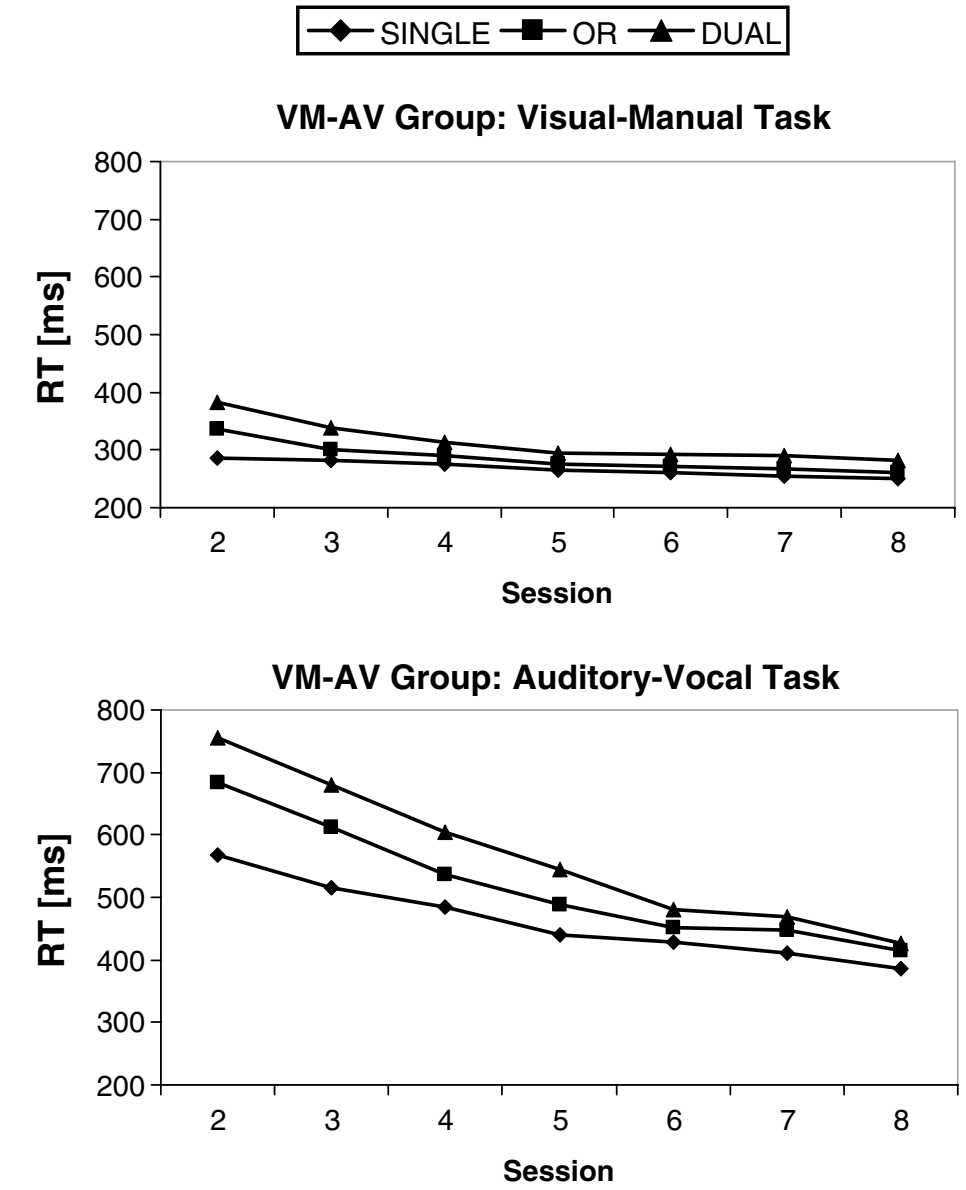

Figure 2. Mean reaction times (RTs) of the VM-AV group of Experiment 1 for the visual task (upper panel) and the auditory task (lower panel). 
TABLE 1

Mean percentage error (PE) with standard errors (in parentheses) for the visual and the auditory tasks of the VM-AV group, for Sessions 2-8 and different trial types (single-task, OR, dual-task) of Experiment 1

\begin{tabular}{llrrrrrrr}
\hline & \multicolumn{8}{c}{ Session } \\
\cline { 3 - 9 } & & \multicolumn{1}{c}{ 2 } & \multicolumn{1}{c}{ 3 } & 4 & 5 & 6 & 7 & 8 \\
\hline \multirow{2}{*}{ Visual Manual } & Single-task & $3.3(0.8)$ & $3.8(0.8)$ & $3.4(1.0)$ & $5.3(1.6)$ & $6.3(1.6)$ & $7.2(1.7)$ & $6.5(1.4)$ \\
& OR & $1.9(0.8)$ & $2.8(0.9)$ & $3.6(1.2)$ & $2.7(1.0)$ & $5.4(2.7)$ & $5.2(2.2)$ & $5.1(1.8)$ \\
& Dual-task & $4.8(1.4)$ & $5.4(2.1)$ & $4.9(1.6)$ & $3.6(1.1)$ & $4.7(1.7)$ & $4.0(1.8)$ & $6.3(2.7)$ \\
Auditory Vocal & Single-task & $6.6(2.4)$ & $6.9(2.1)$ & $8.2(2.7)$ & $7.0(1.7)$ & $8.6(2.2)$ & $8.9(2.1)$ & $7.0(1.5)$ \\
& OR & $7.0(2.4)$ & $7.7(3.3)$ & $6.7(2.0)$ & $6.6(2.0)$ & $8.6(2.6)$ & $8.3(2.6)$ & $6.8(2.0)$ \\
& Dual-task & $12.8(3.7)$ & $12.4(3.9)$ & $11.0(3.8)$ & $9.9(3.0)$ & $11.3(3.5)$ & $10.3(3.1)$ & $10.0(3.0)$ \\
\hline
\end{tabular}

Session $8(32 \mathrm{~ms}), t(7)=2.02, p>.08$ (dual minus single). Dual-task costs from Session 2 to Session 8 were reduced up to a level of nonsignificant $32 \mathrm{~ms}$. Similar results were obtained when comparing dual and OR trials. Here, performance costs were also strongly reduced over practice and fell just short of significance in Session $8(21 \mathrm{~ms})$, $t(7)=2.22, p>.06$ (dual minus OR).

Reaction time analysis of the auditory task. As can be seen in Figure 2 (lower panel), participants responded faster with increasing practice as reflected by a main effect of practice, $F(6$, $42)=45.34, M S E=4669.07, p<.001$. RTs for single-task trials were faster than for OR trials which in turn were faster than for dual-task trials, as reflected by a main effect of trial type, $F(2$, $14)=17.55, \quad M S E=8781.37, p<.001$. Also, a significant interaction of the factors practice $\times$ trial type was observed, $F(12,84)=9.60$, $M S E=720.36, p<.001$, indicating that practice reduced dual-task costs. In Session 2, the comparison between single- and dual-task trials revealed large dual-task costs of $188 \mathrm{~ms}, t(7)=5.26$, $p<.01$, which were drastically reduced to $40 \mathrm{~ms}$, $t(7)=4.15, p<.05$ in Session 8 (dual minus single). A similar reduction was found when comparing dual-task and OR trials. Here, the initial amount of dual-task costs of $72 \mathrm{~ms}$, $t(7)=2.37, p<.05$ in Session 2 was strongly reduced to nonsignificant $11 \mathrm{~ms}$ in Session 8, $t(7)=0.78, p>.45$ (dual minus OR). These results indicate that after eight sessions of practice, dual-task costs were strongly reduced and almost eliminated when these costs were calculated on the basis of OR trials.

Error analysis of the visual task. The same analyses as for RTs were conducted for error rates (see Table 1). Practice did not affect error rates, $F(6,42)=2.89, M S E=8.62, p>.05$. No signifi- cant effect of trial type was observed $(F<1)$. We did not find a significant interaction of practice and trial type, $F(12,84)=2.20, M S E=4.20$, $p>.05$, indicating no reliable effect of practice on different trial types. Despite the lack of statistical effect, we investigated dual-task cost reduction in more detail with planned $t$-tests for Sessions 2 and 8. In Session 2, dual-task and single-task error rates did not differ, $t(7)=1.74$, $p>.12$, but dual-task error rates were slightly higher compared to error rates on OR trials in Session 2, $t(7)=3.24, p<.05$ (see Table 1 ). Most importantly, in Session 8 no difference was observed between dual-task and single-task trials, $t(7)=0.10, p>.9$, and between dual-task and OR trials, $t(7)=0.88, p>.4$.

Error analysis of the auditory task. In the auditory task, an effect of trial type was observed, $F(2,14)=6.25, M S E=38.74, p<.05$, indicating larger error rates in dual-task trials $(11.1 \%)$ as compared to single $(7.6 \%)$ and OR trials $(7.4 \%)$. No interaction of practice $\times$ trial type was observed, $F(12,84)=1.27, \quad M S E=5.52, \quad p>.31$, indicating that the differences in error rates between trial types did not change across practice. In Session 2, more errors were produced in dualtask trials than in single-task trials, $t(7)=3.42$, $p<.05$, and in OR trials, $t(7)=3.26, p<.05$, respectively. After practice in Session 8, no significant difference between dual-task and single-task error rates was observed, $t(7)=1.84$, $p=.11$, but there were still more errors committed in dual-task trials $(10.0 \%)$ as compared to OR trials $(6.8 \%), t(7)=2.86, p<.05$.

Taken together, the analyses of RTs and error rates indicate that dual-task costs were strongly reduced after extensive dual-task practice, but small residual costs remained. 


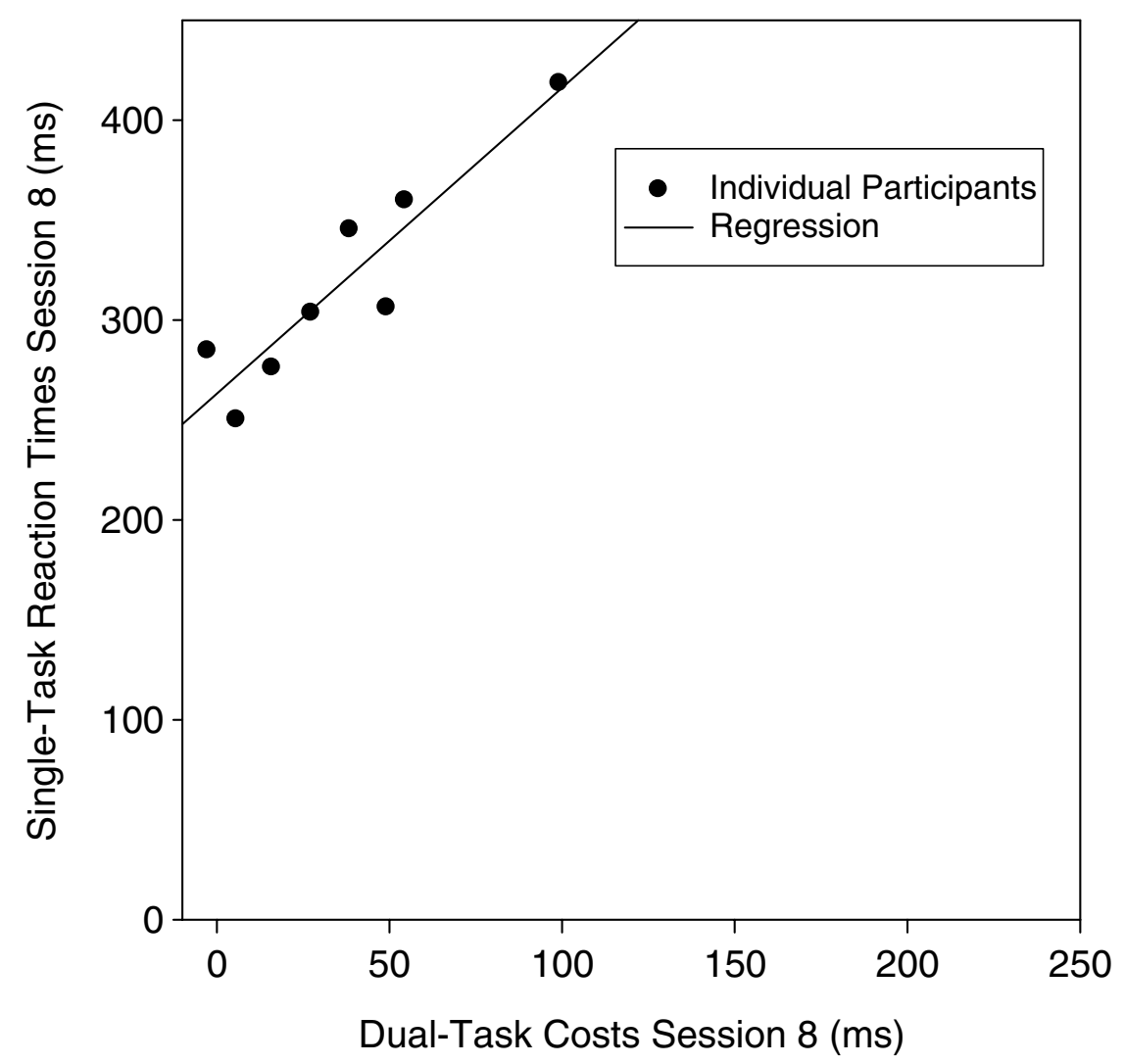

Figure 3. Dual-task costs against single-task RTs in Session 8 averaged across tasks for the eight participants of the VM-AV group in Experiment 1.

\section{Individual differences}

We further investigated systematic individual differences in dual-task cost reduction after practice that emerged in Experiment 1. Figure 3 shows dual-task costs against single-task RTs for each participant in Session 8 averaged across tasks. We split the group with respect to the expected mean in two different subgroups, participants with low interference scores and participants with high interference scores. Analysing dual-task costs in both of these groups indicated that the former exhibit quite small amounts of interference after practice $(17 \mathrm{~ms}), F(1,3)=4.48$, $p=.13$; partial $\eta^{2}=.59$. Two participants came close to zero dual-task costs, qualifying these participants as virtually perfect time sharers. Participants of the latter group showed much larger and robust amounts of interference $(63 \mathrm{~ms})$, $F(1,3)=154.50, p=.001 ;$ partial $\eta^{2}=.98$. Further analyses about individual performance characteristics indicate what may have mediated these differences between subgroups. We found a strong positive correlation of individual dualtask costs and single-task RTs. That is, partici- pants with fast responses in single-task condition in Session 8 also showed low interference scores (dual-task costs) after practice (Session 8), whereas participants with slow responses in the single-task condition showed high interference scores, $r^{2}=.85, p=.001$.

\section{Discussion}

In Experiment 1, we tested if the basic task conditions used in the present study (the use of an equal priority instruction, of a zero SOA, and of a monetary reward system) are sufficient to achieve dual-task cost elimination. We found high dual-task costs before practice, which were extremely reduced by the end of learning. However, we did not find the conservative criteria of zero dual-task costs for RTs and error rates after practice (Schumacher et al., 2001). Relatively small residual dual-task costs remained, which is in line with the findings of previous studies (Hazeltine et al., 2002; Tombu \& Jolicoeur, 2004; van Selst et al., 1999). Our findings suggest that the present task conditions can be considered 
as sufficient to achieve high levels of dual-task cost reduction over practice. Interestingly, we found relatively small mean single-task RTs after eight sessions of practice suggesting that our deadline procedure using separate deadlines in single-task and dual-task conditions (Tombu \& Jolicoeur, 2004) boosted motivation in both, single-task and dual-task conditions. This might have counteracted a complete dual-task cost reduction in the present study compared to the findings of Schumacher et al. (2001).

The analyses of our data on an individualparticipants level revealed a deeper insight on the finding of dual-task cost reduction. These analyses qualified some participants as virtually perfect time sharers showing complete dual-task cost reduction, whereas three out of eight participants showed costs of more than $50 \mathrm{~ms}$ after practice. A good predictor for dual-task cost reduction was the single-task performance in Session 8. In other words, the faster participants in single-task responses after practice, the smaller their residual dual-task costs. The fact that some participants were able to fully reduce their dualtask costs and others not could theoretically be explained by strategic bottleneck models (Meyer \& Kieras, 1997a, 1997b), under which participants may use a variety of task-scheduling strategies (from cautious to daring task processing). However, the strong correlation we found between single-task response times and the amount of dual-task costs that was achieved after practice is also in line with the predictions made by a latent bottleneck model (Ruthruff et al., 2003) explaining individual differences in dual-task cost reduction with variations in single-task response times.

\section{EXPERIMENT 2}

In Experiment 2, we tested if dual-task costs can be eliminated or at least greatly reduced (as observed in Experiment 1) in a VM-AP dualtask situation using a non-PRP design. We applied the Meyer and Kieras (1997a, 1997b) criteria for dual-task cost reduction as used in Experiment 1 to the VM-AP task of Experiment 2. In line with Sangals et al. (2007), we distributed the visual stimuli to both hands and the auditory stimuli to both feet. Crosstalk effects (Hommel, 1998; Koch \& Prinz, 2002; Lien \& Proctor, 2002) due to the strong left-right dimension in both tasks might hinder dual-task cost reduction under incompatible conditions (e.g., left-hand and right- foot response), but might help to reduce dual-task costs under compatible R-R conditions (e.g., lefthand and left-foot response). We aim to test if dual-task cost elimination can be achieved under compatible dual-task conditions, when both tasks involve the activation of the same dimension, such as left-left. To test this, we created a strong dimensional left-right overlap in the VM-AP task pairing.

\section{Methods}

Participants. Eight new undergraduate students (2 male, age: 19-26, mean age: 22.0 years) of Humboldt-University, Berlin, took part in this experiment. All participants had normal or corrected-to-normal vision, and were naive about the hypotheses of the experiment. Participants gave their written informed consent to participate in the study, which was conducted in accordance with the ethical standards of the 1964 Declaration of Helsinki.

Apparatus and stimuli. In the VM-AP condition, two red circles served as placeholders in the VM task. One of the circles changed its colour from red to green. Participants responded manually to the circle that changed its colour (red to green) in a spatially compatible mapping with a keypress using the left index or the right index finger, respectively. The circles were arranged horizontally on a black background of a computer monitor. Each circle subtended approximately $1.1 \mathrm{~cm}$, which corresponds to $1.02^{\circ}$ of visual angle at a viewing distance of $60 \mathrm{~cm}$. The two circles were separated by $0.3 \mathrm{~cm}$, approximately $0.28^{\circ}$ of visual angle. The circles spread over a distance of $2.38^{\circ}$. The circles remained on the screen until the participant responded or until $2000 \mathrm{~ms}$ had expired. The responses were recorded with a response board connected to the computer.

For the auditory task, participants responded with their left foot to a low tone $(350 \mathrm{~Hz})$ and with their right foot to a high tone $(1650 \mathrm{~Hz})$. The tones were played on headphones for $40 \mathrm{~ms}$.

Procedure and design. The procedure and design was identical to Experiment 1.

\section{Results}

Reaction time analysis of the visual task. Prior to statistical RT analyses, all trials in which responses were incorrect were excluded (3.9\%). 
Data of Experiment 2 were subject to the same analyses as in Experiment 1. The results are presented in Figure 4, upper panel. Participants responded faster with increasing practice, $F(6$, $42)=29.52, \quad M S E=1568.28, \quad p<.001$. RTs in dual-task trials were slower than in $\mathrm{OR}$ and single-task trials as indicated by a main effect of trial type, $F(2,14)=68.05, M S E=9038.94$, $p<.001$. We observed a significant interaction of the factors practice and trial type, $F(12$, $84)=15.25, M S E=767.05, p<.001$. That is, practice reduced dual-task costs from $344 \mathrm{~ms}$, $t(7)=7.96, p<.001$, in Session 2 to $126 \mathrm{~ms}$ in Session $8, t(7)=5.85, p<.01$ (dual minus single). A similar dual-task cost reduction from $232 \mathrm{~ms}$, $t(7)=6.70, p<.001$, in Session 2 to $96 \mathrm{~ms}$, $t(7)=5.47, p<.01$, in Session 8 was found when comparing dual-task and OR trials respectively (dual minus OR).

Practice improved dual-task costs in the visual task. However, a high level of dual-task costs
(126 ms) remained even after eight sessions of practice.

Reaction time analysis of the auditory task. Results are presented in Figure 4 (lower panel). Participants responded faster with an increasing amount of practice, as reflected in the main effect of practice, $F(6,42)=28.58, M S E=2387.07$, $p<.001$. Dual-task trials were slower than OR trials, which in turn were slower than single-task trials, as indicated by a main effect of trial type, $\quad F(2, \quad 14)=137.51, \quad M S E=7497.62$, $p<.001$. Further, we observed a significant interaction of the factors practice and trial type, $F(12,84)=15.59, M S E=576.08, p<.001$, showing that dual-task costs were reduced over practice. In Session 2, we found dual-task costs in the auditory task amounting to $394 \mathrm{~ms}, t(7)=11.01$, $p<.001$ (dual minus single). RTs in dual-task and OR trials differed in Session 2 by $162 \mathrm{~ms}$, $t(7)=7.59, p<.001$ (dual minus OR). After eight

\section{$\bullet$ SINGLE $\rightarrow-\mathrm{OR} \rightarrow-\mathrm{DUAL}$}
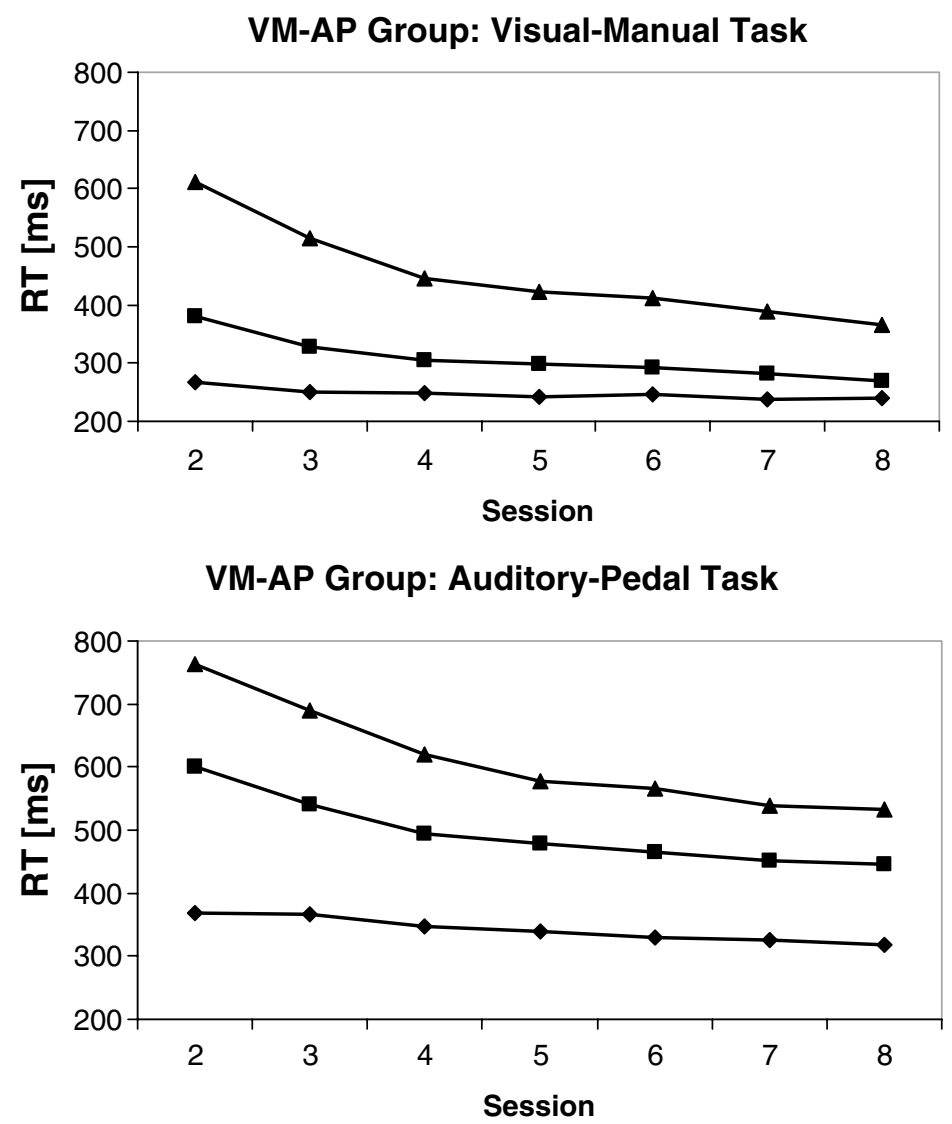

Figure 4. Mean reaction times (RTs) of the VM-AP group of Experiment 2 for the visual task (upper panel) and the auditory task (lower panel). 
sessions of practice, dual-task costs remained high amounting to $216 \mathrm{~ms}, t(7)=9.32, p<.001$, when comparing single-task and dual-task trials (dual minus single). Performance in dual-task trials was also impaired compared to that in OR trials in Session 8 by $88 \mathrm{~ms}, t(7)=6.21, p<.001$ (dual minus OR). Thus, similar to the visual task, dual-task costs remained large $(216 \mathrm{~ms})$ after eight sessions of practice.

Error analysis of the visual task. Error rates increased with practice from Session $2(2.5 \%)$ to Session $8(4.7 \%), F(6,42)=6.24, M S E=2.30$, $p<.001$. More errors were performed in single tasks $(8.2 \%)$ than in OR $(1.4 \%)$ and dual-task trials $(1.3 \%)$, as shown by an effect of trial type, $F(2,14)=12.02, M S E=74.06, p<.01$. Although error rates were reduced in dual-task trials over practice, we found an increase in single-tasks error rates, as indicated by a significant interaction of practice and trial type, $F(12,84)=5.18$, $M S E=2.69, p<.001$. In Session 2, error rates between dual-task and single-task trials did not differ statistically, $t(7)=0.69, p>.5$, but $2.7 \%$ more errors were committed in dual-task trials compared to OR trials, $t(7)=5.74, p<.01$ (see Table 2). In Session 8 participants performed $8.7 \%$ more errors in single-task trials than in dual-task trials, $t(7)=4.89, p<.01$. No significant difference for error rates was observed between dual-task trials and OR, $t(7)=1.46, p>.18$.

Error analysis of the auditory task. In the auditory task, no significant effect of practice was observed $(F<1)$, indicating that practice did not affect error rates (see Table 2 ). We observed an effect of trial type, $F(2,14)=5.16$, $M S E=14.01, p<.05$, indicating higher error rates in dual-task trials $(4.8 \%)$ than in OR trials $(2.6 \%)$ and in single-task trials $(4.2 \%)$. We found a significant interaction of practice and trial type, $F(12,84)=3.46, M S E=8.09, p<.05$. Participants performed $4.1 \%$ more errors in dual-task trials as compared to single-task trials in Session 2, $t(7)=3.82, p<.01$. They also performed $4.6 \%$ more errors in dual-task trials as compared to OR trials, $t(7)=5.26, p<.01$. In Session 8 error rates in dual-task trials were reduced so that they did not differ when compared to single-task trials. Taken together, dual-task costs were not eliminated but instead remained on a relatively high level after practice in the VM-AP task pairing.

\section{Individual differences}

We also tested for systematic individual differences in dual-task performance that emerged in Experiment 2. Figure 5 shows dual-task costs in Session 8 against single-task RTs of Session 8 averaged across both tasks. A group split with respect to the expected mean resulted in two subgroups, participants with relatively low interference scores and those with high interference scores. Participants of the low interference group showed $187 \mathrm{~ms}$ dual-task costs after eight sessions of practice, $F(1,3)=103.36, p=.002$; partial $\eta^{2}=.97$. Participants of the high interference group showed dual-task costs of $246 \mathrm{~ms}, F(1$, $3)=37.96, p=.009$; partial $\eta^{2}=.93$. In addition, we found no correlation between dual-task costs and single-task RTs that was achieved after practice, $r^{2}=.12, p=.39$. No single participant was able to reduce his/her dual-task costs after practice not even approximately to zero. The highest level of dual-task cost reduction that was reached by one participant was a reduction to $120 \mathrm{~ms}$ of dual-task costs after practice.

TABLE 2

Mean percentage error (PE) with standard errors (in parentheses) for the visual and the auditory tasks of the VM-AP group, for Sessions 2-12 and different trial types (single-task, OR, dual-task) of Experiment 2

\begin{tabular}{|c|c|c|c|c|c|c|c|c|c|c|c|c|}
\hline & & \multicolumn{11}{|c|}{ Session } \\
\hline & & 2 & 3 & 4 & 5 & 6 & 7 & 8 & 9 & 10 & 11 & 12 \\
\hline \multirow{3}{*}{$\begin{array}{l}\text { Visual } \\
\text { Manual }\end{array}$} & Single-task & $4.3(2.0)$ & $6.9(2.7)$ & $9.2(2.3)$ & $8.8(2.4)$ & $9.3(2.2)$ & $9.0(1.8)$ & $10.2(1.8)$ & $9.2(1.1)$ & $8.0(1.2)$ & $11.3(1.5)$ & $9.4(1.0)$ \\
\hline & OR & $0.2(0.1)$ & $0.5(0.4)$ & $1.0(0.5)$ & $1.7(0.8)$ & $1.5(0.6)$ & $2.4(0.6)$ & $2.5(0.7)$ & $3.0(0.7)$ & $3.1(0.6)$ & $3.1(0.4)$ & $2.6(0.5)$ \\
\hline & Dual-task & $2.9(0.5)$ & $0.9(0.2)$ & $1.1(0.5)$ & $0.9(0.3)$ & $0.8(0.2)$ & $0.9(0.2)$ & $1.5(0.2)$ & $1.4(0.4)$ & $1.6(0.4)$ & $1.4(0.3)$ & $1.0(0.4)$ \\
\hline \multirow{3}{*}{$\begin{array}{c}\text { Auditory } \\
\text { Pedal }\end{array}$} & Single-task & $3.0(1.5)$ & 4.1(1.7) & $3.3(1.1)$ & $4.3(1.4)$ & $4.9(1.7)$ & $4.6(1.1)$ & $5.1(1.3)$ & $6.3(1.8)$ & $6.3(1.6)$ & $5.5(1.6)$ & $6.9(1.9)$ \\
\hline & OR & $2.4(0.8)$ & $2.5(1.0)$ & 2.1(0.6) & $2.7(0.9)$ & $2.1(0.7)$ & $2.7(0.8)$ & $3.8(1.3)$ & $3.5(0.9)$ & $2.4(0.8)$ & $3.2(0.7)$ & $3.5(1.2)$ \\
\hline & Dual-task & 7.1(1.5) & $5.4(1.5)$ & $4.2(1.0)$ & 5.1(1.3) & $3.8(1.2)$ & $3.9(1.2)$ & $4.4(1.2)$ & $3.4(1.0)$ & $4.3(1.5)$ & $5.0(1.2)$ & $5.4(1.2)$ \\
\hline
\end{tabular}

$\mathrm{VM}=$ visual-manual; AP = auditory-pedal. 


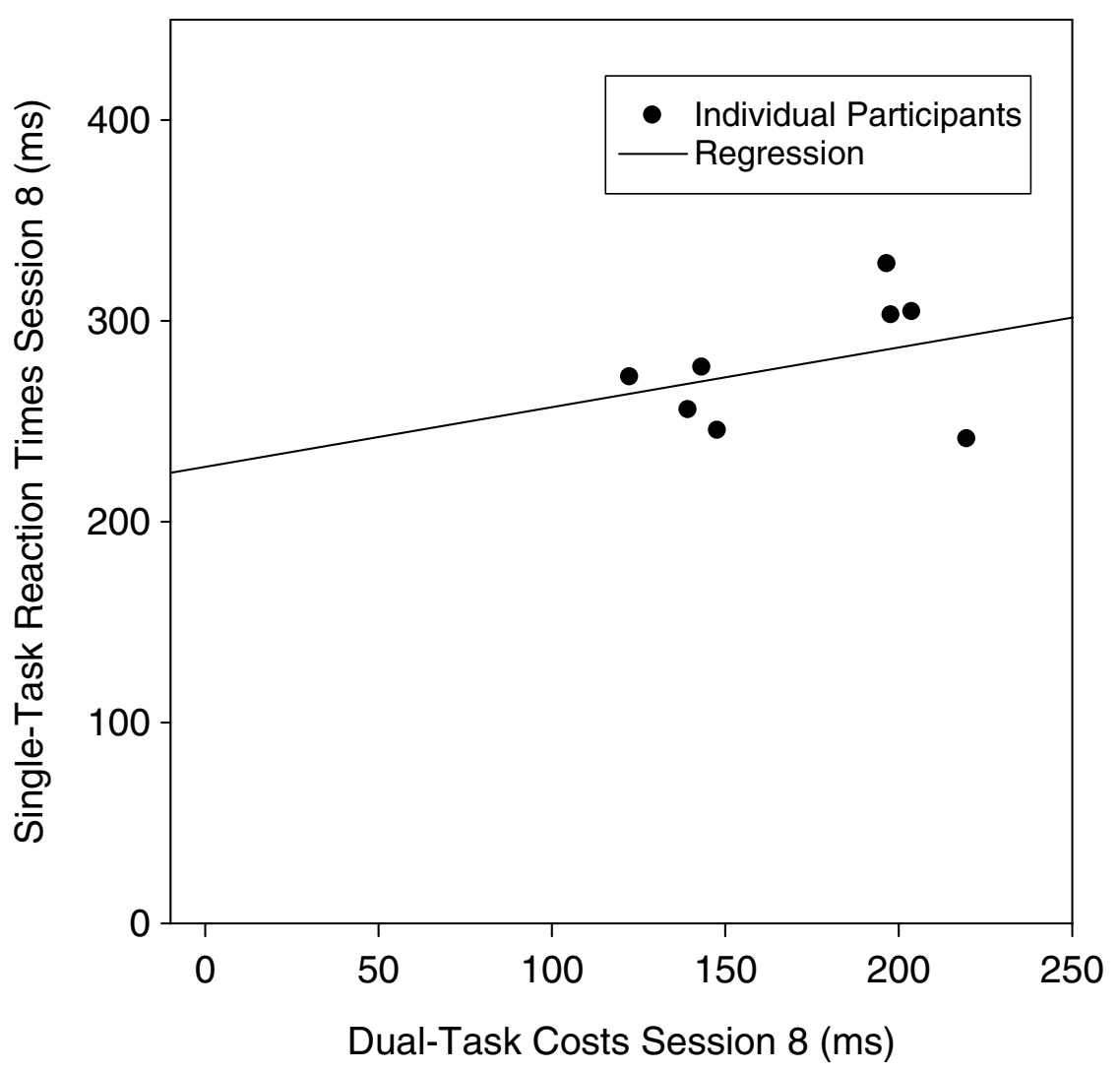

Figure 5. Dual-task costs against single-task RTs in Session 8 averaged across tasks for the eight participants of the VM-AP group in Experiment 2.

\section{Extended practice}

Because in Experiment 2 we found relatively large remaining dual-task costs at the end of Session 8 , we decided to increase the amount of practice by asking the same participants to perform four additional practice sessions (Sessions 9-12). The prolongation of practice allowed us to assess whether participants were able to achieve a sufficient level of dual-task performance, i.e., further cost reduction. We first analysed whether participants reached a learning plateau during their prolonged practice by comparing performance across the final three learning sessions. For that purpose, we performed a $3 \times 3$-factorial ANOVA including the factors practice (Sessions 10-12) and trial type.

Visual task. We found no further improvement during prolonged practice, as indicated by a nonsignificant effect of practice, $F(2,14)=1.85$, $M S E=112.34, p>.19$. The dual-task costs observed in Session 8 persisted even after prolonged practice, as indicated by a main effect of trial type,
$F(2,14)=38.9, M S E=2455.51, p<.001$, and a lacking interaction effect of practice and trial type $F(4,28)=1.23, M S E=48.86, p>.3$, in the VMAP condition.

Auditory task. As with the visual task, we found no significant effect of practice, $F(2,14)=2.48$, $M S E=870.43, p>1$. Dual-task costs remained stable even after prolonged practice as shown by an effect of trial type, $F(2,14)=45.7$, $M S E=3668.41, p<.001$. Increasing the amount of practice from 8 to 12 sessions did not change the pattern of results as indicated by a nonsignificant interaction of practice and trial type in the auditory task, $F(4,28)=1.20, M S E=226.56$, $p>$.3. These findings suggest that a learning plateau had been reached in both tasks by the end of Session 8.

In order to strengthen the claim that dual-task costs remain during prolonged practice we additionally analysed the learning rate across the last three learning sessions by comparing ratios (raw dual-task RTs/single task RTs) for both tasks. If the ratios do not differ across sessions during 
extended practice, then, we assume similar learning rates for single- and dual-task trials which cause dual-task costs to remain constant across these sessions. We performed a three-factorial ANOVA including the within-subjects variable practice (Sessions 10-12) for the learning rates separately for each task. Importantly, the effect of additional practice on the learning rate was neither significant for the visual task, $F(2$, $14)=1.10, M S E=0.005, p>.34$ (learning rates: Session $10=1.49$, Session $11=1.53$, Session $12=1.49)$, nor for the auditory task, $F(2$, 14) $=1.56, \quad M S E=.008, \quad p>.24 \quad$ (Session $10=1.59$, Session $11=1.51$, Session $12=1.54$ ).

\section{Discussion}

In Experiment 2, we tested if dual-task costs can be eliminated in a VM-AP dual-task situation using a non-PRP design applying the Meyer and Kieras (1997a, 1997b) criteria for time sharing. We found large dual-task costs before practice, which were reduced after 8 hours of practice. However, dual-task costs remained on a high level and were far from close to elimination. Errors in the single-task condition seem to increase with practice. This finding suggests that participants were motivated to perform at their performance limits. When there is not much room for improvement but a high motivation to further improve task performance, an error increase in single-task performance is likely. This finding also indicates the importance of a motivation procedure taking into account both, single-task and dual-task conditions. Individual-subject analyses showed that none of the participants revealed dual-task cost reductions that came close to perfect time sharing. This result is substantiated by a lack of correlation between single-task performance and the amount of dual-task cost reduction at the end of learning, which suggests that learning did not lead to latent bottlenecks.

\section{GENERAL DISCUSSION}

Previous studies showed resisting dual-task costs over practice using VM-AP task pairings (Sangals et al., 2007) which are often used in humanmachine interfaces. The aim of the present study was to test if dual-task costs can be eliminated during practice with a VM-AP task when using non-PRP task conditions and applying a set of criteria that have mostly been selected to achieve perfect time sharing (Meyer \& Kieras, 1997a, 1997b) and which have effectively been used in previous studies (Hazeltine et al., 2002; Schumacher et al., 2001). These criteria concern the use of an equal priority instruction, a zero SOA, and a monetary reward system. To test the efficiency of these criteria, we first conducted a VM-AV control condition in Experiment 1 using essentially the same task conditions as in previous studies that showed evidence for time sharing (Hazeltine et al., 2002; Schumacher et al., 2001). In this condition we found high dual-task costs before practice and a great reduction of dual-task costs. However, small residual dual-task costs remained after practice. The present findings replicate the results of a range of previous studies (Hazeltine et al., 2002; Tombu \& Jolicoeur, 2004) showing that the present design seems to be sufficient for achieving a high level of dual-task cost reduction. However, we did not find evidence for complete dual-task cost elimination in both tasks and all participants, as found in the Schumacher et al. (2001) study. The fact that RTs in the single-task condition were small after practice might have counteracted a full reduction of dualtask costs in Experiment 1. Indeed, RTs in the corresponding visual single task of the Schumacher et al. (2001) study were slower than those obtained in the present study. This finding could be explained by using separate deadlines for dualtask and single-task conditions in the present study, which keeps a constant high motivation equally for both single-tasks and dual-tasks till the end of practice (Tombu \& Jolicoeur, 2004). We further found large interindividual differences in dual-task performance, which manifests in both differences in single-task performance and the amount of dual-task cost reduction that was achieved after practice: Short single-task RTs in Session 8 go together with a large amount of dualtask cost reduction after practice, qualifying some participants as perfect time sharers, but leave others with residual dual-task costs of more than $50 \mathrm{~ms}$ after practice. Individual differences in the amount of dual-task cost reduction that was achieved over practice could come from a variation of single-task response times. In line with this assumption, we found a close correlation between dual-task costs and single-task performance that was achieved after practice. These findings are in line with the assumption that a bottleneck remains latent after practice (Ruthruff et al., 2003). 
In Experiment 2, we used a VM-AP dual-task combination similar to the one used in the Sangals et al. (2007) study, but using a non-PRP dual-task situation, as in Experiment 1. We also adapted task instructions and task conditions to those that have effectively been used in Experiment 1 and a range of previous studies (Hazeltine et al., 2002; Schumacher et al., 2001; Tombu \& Jolicoeur, 2004) to achieve great levels of dualtask cost reduction after practice. Importantly, our results showed that large dual-task costs were found at the beginning of practice, which declined substantially by 8 hours of training. Participants in Experiment 2, however, did not achieve complete dual-task cost reduction. Even though participants in Experiment 2 may use a variety of taskscheduling strategies, not even the person that may have adopted the most optimal task-scheduling strategy was able to approximately reach the level of perfect time sharing. In the VM-AP dualtask combination the best performance that was reached after 8 hours of practice was over $120 \mathrm{~ms}$ dual-task costs. Individual participants' analyses showed no correlation between dual-task costs and single-task performance after practice. Robust dual-task costs found in Experiment 2 were also not simply a matter of practice duration. High and enduring dual-task costs remained in the VM-AP setting even after further extending practice. Dual-task costs were especially high for the auditory task. RT analyses suggest that dualtask costs had reached a plateau during extended practice. The remaining dual-task costs seem to be relatively resistant to further improvement. These findings were also confirmed by a direct comparison of dual-task costs across experiments which showed significantly higher dual-task costs after 8 hours of practice in the VM-AP group as in the VM-AV group, both in the visual-task $(p<.05)$, as well as in the auditory task $(p<.01)$. This finding further strengthens the claim that dual-task cost reduction in the VM-AP group (Experiment 2) is much more resistant against practice effects than in the VM-AV group (Experiment 1). These findings suggest that the resistance of dual-task cost reduction in the VM-AP group is not only a matter of applying the same criteria as argued by Schumacher et al. (2001) to be relevant to reach complete dual-task cost reduction. Applying these criteria seems not sufficient to circumvent a bottleneck in the VMAP group. Therefore other sources of interference need to be considered. The major candidate hypotheses for the large residual dual-task costs in the VM-AP group are (1) crosstalk between the response codes of the two tasks prevents dualtask cost reduction and (2) the specific input and output modality pairings produce additional interference between the central processes of the two tasks.

\section{Persisting dual-task costs in the VM-AP group due to crosstalk?}

The finding of persisting dual-task costs over practice in the VM-AP group replicates and also extends previous studies (Sangals et al., 2007). Sangals et al. (2007) reported high and relatively resistant dual-task costs after five practice sessions using a PRP paradigm (Pashler, 1994). Even when the criteria guidelines of the VM-AP group of Experiment 2 closely follow those used in the VM-AV task setting of Experiment 1, as well as in other studies (Hazeltine et al., 2002; Schumacher et al., 2001) we still did not find evidence for dualtask cost elimination and time sharing.

Some specific properties of hand and foot pairings, in the way they were used in the present study might open up ways to understand what might have prevented dual-task cost reduction over practice: One possibility may be found in the spatial dimensional overlap of response codes of the two tasks. Using manual and pedal output modalities with distributed responses might introduce crosstalk effects, on the basis of spatially mediated R-R compatibility (e.g., Hommel, 1998; Koch \& Prinz, 2002; Lien \& Proctor, 2002; Logan \& Gordon, 2001; Schubert et al., 2008). In line with theories highlighting the role of contentbased interference in dual-task processing (Navon \& Miller, 1987) this kind of crosstalk might be especially pronounced in the VM-AP task condition in which both effectors involve primary spatial dimensional attributes. More recent findings of Lien and colleagues suggest that the cognitive system might simultaneously activate multiple responses relating to multiple stimuli (Lien, Ruthruff, Hsieh, \& Yu, 2007). Interference effects due to a failure of selective task activation are most likely with strong dimensional overlap between tasks, so that the stimuli or responses of one task activate the other task as well. In the present study, the stimuli of the VM task correspond to the responses of the AP task, increasing the likelihood of failures in selective task activation. The stimulus for the VM task is highly 
compatible with the response for the AP task, tempting the participants to use the VM stimulus to guide the AP response which might lead to facilitation effects for compatible task effector pairings (e.g., left-left responses), but interference effects for incompatible pairings (left-right responses). To test if dual-task costs are fully reduced in the VM-AP condition for compatible condition, we compared the dual-task performance on compatible trials with the single-task performance. Taking only compatible dual-task trials into account, we still observed residual dualtask costs of $102 \mathrm{~ms}(p<.05)$ in the visual task and of $121 \mathrm{~ms}(p<.05)$ in the auditory task. This finding suggest that even though crosstalk seems a relevant factor with respect to the amount of dual-task costs that can be achieved with practice it might not fully explain the large residual dualtask costs that remained in the VM-AP group after practice.

\section{VM-AP a nonstandard modality pairing?}

Findings of a recent study (Hazeltine et al., 2006) testing potential sources of dual-task interference compared the amount of dual-task cost reduction when the typical input and output modality pairings (VM-AV) of Schumacher et al. (2001) were paired in a nonstandard manner (i.e., VVAM). They found much larger and more resistant dual-task costs after extended practice for nonstandard task pairings as for standard pairings. Hazeltine et al. (2006) concluded that differences in dual-task costs at the end of learning between conditions are due to content-dependent interference between the central processes for the two tasks, which is especially high for nonstandard task pairings (see also Hazeltine \& Ruthruff, 2006; Stelzel, Schumacher, Schubert, \& D'Esposito, 2006). The VM-AP task pairing used in the present study may also represent such a nonstandard modality pairing, which could potentially explain remaining dual-task costs that can not be explained by crosstalk.

The present findings have important implications for the literature on dual-task learning. Our findings in the VM-AV setting of Experiment 1 partially replicate Schumacher et al.'s (2001) results. Some participants showed evidence for time sharing in the VM-AV condition of Experiment 1 after 8 hours of practice. At the same time, the findings of the VM-AP condition in Experiment 2 limit the generalisation of the Schumacher results and suggest that the amount of dual-task cost reduction critically depends on the specific task combination and task conditions. No single participant showed evidence for time sharing in the present VM-AP pairing of Experiment 2. Despite nonoverlapping perceptual and effector systems (Meyer \& Kieras, 1997a; see also Allport, Antonis, \& Reynolds, 1972; Neumann, 1987) and the careful consideration of the criteria proposed as optimal for dual-task cost reduction (Meyer \& Kieras, 1997a, 1997b) dual-task costs were not eliminated in the VM-AP condition of Experiment 2. These findings suggest that additional sources of interference contribute to the finding of remaining dual-task costs after practice.

\section{CONCLUSION}

The present study shows that the finding of resistant dual-task costs after prolonged practice (Sangals et al., 2007) is not simply due to the paradigm that was used, to arranging for Task 1 priority, to the duration of practice, or to a lack of monetary reward. Applying the same criteria as argued by Schumacher et al. (2001) to be conducive for reaching complete dual-task cost reduction, we obtained no evidence for time sharing. These results suggest that Schumacher et al.'s finding of complete dual-task cost reduction does not generalise to the VM-AP task combination used in the present study.

\footnotetext{
Original manuscript received April 2009

Revised manuscript received October 2009
}

\section{REFERENCES}

Allport, D. A., Antonis, B., \& Reynolds, P. (1972). On the division of attention: A disproof of the singlechannel hypothesis. Quarterly Journal of Experimental Psychology, 24, 225-235.

Beringer, J. (1987-2000). Experimental Runtime System (Software). BeriSoft Corporation, Frankfurt am Main, Germany.

Byrne, M. D., \& Anderson, J. R. (2001). Serial modules in parallel: The psychological refractory period and perfect time-sharing. Psychological Review, 108, 847-869.

De Jong, R. (1993). Multiple bottlenecks in overlapping task performance. Journal of Experimental Psychology: Human Perception and Performance, 19, 965980. 
Hazeltine, E., \& Ruthruff, E. (2006). Modality pairing effects and the response selection bottleneck. Psychological Research, 70, 504-513.

Hazeltine, E., Ruthruff, E., \& Remington, R. W. (2006). The role of input and output modality pairings in dual-task performance: Evidence for content-dependent central interference. Cognitive Psychology, 52, 291-345.

Hazeltine, E., Teague, D., \& Ivry, R. (2002). Simultaneous dual-task performance reveals parallel response selection after practice. Journal of Experimental Psychology: Human Perception and Performance, 28, 527-545.

Hirst, W., Spelke, E. S., Reaves, C. C., Caharack, G., \& Neisser, U. (1980). Dividing attention without alternation or automaticity. Journal of Experimental Psychology: General, 109, 98-117.

Hommel, B. (1998). Automatic stimulus-response translation in dual-task performance. Journal of Experimental Psychology: Human Perception and Performance, 24, 1368-1384.

Karlin, L., \& Kestenbaum, R. (1968). Effects of number of alternatives on the psychological refractory period effect. Quarterly Journal of Experimental Psychology, 20, 167-178.

Keele, S. (1973). Attention and human performance. Palisades, CA: Goodyear.

Koch, I., \& Prinz, W. (2002). Process interference and code overlap in dual-task performance. Journal of Experimental Psychology: Human Perception and Performance, 28, 192-201.

Lien, M. C., \& Proctor, R. W. (2002). Stimulusresponse compatibility and psychological refractory period effects: Implications for response selection. Psychonomic Bulletin and Review, 9, 212-238.

Lien, M. C., Ruthruff, E., Hsieh, S., \& Yu, Y. T. (2007). Parallel central processing between tasks: Evidence from lateralized readiness potential. Psychonomic Bulletin and Review, 14, 133-141.

Logan, G. D., \& Gordon, R. D. (2001). Executive control of visual attention in dual-task situations. Psychological Review, 108, 393-434.

Meyer, D. E., \& Kieras, D. E. (1997a). A computational theory of executive cognitive processes and multiple-task performance: Part 1. Basic mechanisms. Psychological Review, 104, 3-65.

Meyer, D. E., \& Kieras, D. E. (1997b). A computational theory of executive cognitive processes and multiple-task performance: Part 2. Accounts of psychological refractory-period phenomena. Psychological Review, 104, 749-791.

Meyer, D. E., \& Kieras, D. E. (1999). Précis to a practical unified theory of cognition and action: Some lessons from EPIC computational models of human multiple-task performance. In D. Gropher \& A. Koriat (Eds.), Attention and performance XVII. Cognitive regulation of performance: Interaction of theory and application (pp. 17-88). Cambridge, MA: MIT Press.

Navon, D., \& Miller, J. (1987). Role of outcome conflict in dual-task interference. Journal of Experimental Psychology: Human Perception and Performance, 13, 435-448.

Neumann, O. (1987). Beyond capacity: A functional view of attention. In H. Heuer \& A. F. Sanders (Eds.), Perspectives on perception and action (pp. 361-394). Hillsdale, NJ: Lawrence Erlbaum Associates, Inc.

Pashler, H. (1994). Dual-task interference in simple tasks: Data and theory. Psychological Bulletin, 116, 220-244.

Pashler, H., \& Johnston, J. C. (1989). Chronometric evidence for central postponement in temporally overlapping tasks. Quarterly Journal of Experimental Psychology, 41A, 19-45.

Ruthruff, E., Johnston, J. C., van Selst, M., Whitsell, S., \& Remington, R. W. (2003). Vanishing dual-task interference after practice: Has the bottleneck been eliminated or is it merely latent? Journal of Experimental Psychology: Human Perception and Performance, 29, 280-289.

Sangals, J., Wilwer, M., \& Sommer, W. (2007). Localising practice effects in dual-task performance. Quarterly Journal of Experimental Psychology, 60, 860876.

Schubert, T. (1999). Processing differences between simple and choice reactions affect bottleneck localization in overlapping tasks. Journal of Experimental Psychology: Human Perception and Performance, 25, 408-425.

Schubert, T., Fischer, R., \& Stelzel, C. (2008). Response activation in overlapping tasks and the response selection bottleneck. Journal of Experimental Psychology: Human Perception and Performance, 34, 376-397.

Schumacher, E. H., Seymour, T. L., Glass, J. M., Kieras, D. E., \& Meyer, D. E. (2001). Virtually perfect time sharing in dual-task performance: Uncorking the central attentional bottleneck. Psychological Science, 12, 101-108.

Spelke, E., Hirst, W., \& Neisser, U. (1976). Skills of divided attention. Cognition, 4, 215-230.

Stelzel, C., Schumacher, E. H., Schubert, T., \& D'Esposito, M. (2006). The neural effect of stimulus-response modality compatibility on dual-task performance: An fMRI study. Psychological Research, 70, 514-525.

Tombu, M., \& Jolicoeur, P. (2004). Virtually no evidence for virtually perfect time-sharing. Journal of Experimental Psychology: Human Perception and Performance, 30, 795-810.

Van Selst, M., Ruthruff, E., \& Johnston, J. C. (1999). Can practice eliminate the psychological refractory period effect? Journal of Experimental Psychology: Human Perception and Performance, 25, 1268-1283. 